\title{
NOTE ON LINEAR TRANSFORMATIONS OF $n$-ICS IN $m$ VARIABLES*
}

BY A. D. CAMPBELL

Let us consider the $n$-ic in $m$ variables

$$
F\left(x_{1}, x_{2}, \cdots, x_{m}\right)=0 .
$$

If we subject (1) to the linear transformation

$$
\begin{aligned}
\rho x_{1} & =a_{11} x_{1}^{\prime}+a_{12} x_{2}^{\prime}+a_{13} x_{3}^{\prime}+\cdots+a_{1 m} x_{m}^{\prime}, \\
\rho x_{2} & =a_{21} x_{1}^{\prime}+a_{22} x_{2}^{\prime}+\cdots+a_{2 m} x_{m}^{\prime}, \cdots, \\
\rho x_{m} & =a_{m 1} x_{1}^{\prime}+a_{m 2} x_{2}^{\prime}+a_{m 3} x_{3}^{\prime}+\cdots+a_{m m} x_{m}^{\prime},
\end{aligned}
$$

we obtain

$$
\begin{aligned}
& F\left(a_{11} x_{1}^{\prime}+a_{12} x_{2}^{\prime}+a_{13} x_{3}^{\prime}+\cdots+a_{1 m} x_{m}^{\prime}, a_{21} x_{1}^{\prime}+a_{22} x_{2}^{\prime}\right. \\
& \quad+a_{23} x_{3}^{\prime}+\cdots+a_{2 m} x_{m}^{\prime}, \cdots, a_{m 1} x_{1}^{\prime}+a_{m 2} x_{2}^{\prime} \\
& \left.\quad+a_{m 3} x_{3}^{\prime}+\cdots+a_{m m} x_{m}^{\prime}\right)=0 .
\end{aligned}
$$

Note that in the expansion of (3) the coefficient of the term in $x_{i}^{\prime n},(i=1,2,3, \cdots, m)$, is $F\left(a_{1 i}, a_{2 i}, a_{3 i}, \cdots, a_{m i}\right)$. A necessary and sufficient condition for this coefficient to vanish is that the point $P_{i}\left(a_{1 i}, a_{2 i}, \cdots, a_{m i}\right)$ shall lie on the geometric locus of (1). To obtain the coefficient of such a term as $x_{i}^{\prime}{ }^{r} x_{j}^{\prime}{ }^{n-r}$ in the expansion of (3) we can put

$$
\begin{aligned}
x_{i}^{\prime} x_{j}^{\prime} & \neq 0, x_{1}^{\prime}=x_{2}^{\prime}=x_{3}^{\prime}=\cdots=x_{i-1}^{\prime} \\
& =x_{i+1}^{\prime}=x_{i+2}^{\prime}=\cdots=x_{j-1}^{\prime}=x_{j+1}^{\prime}=\cdots=x_{m}^{\prime}=0,
\end{aligned}
$$

then use Taylor's Expansion on

$$
\begin{aligned}
& F\left(a_{1 i} x_{i}^{\prime}+a_{1 j} x_{j}^{\prime}, a_{2 i} x_{i}^{\prime}+a_{2 j} x_{j}^{\prime}, a_{3 i} x_{i}^{\prime}\right. \\
& \left.\quad+a_{3 j} x_{j}^{\prime}, \cdots, a_{m i} x_{i}^{\prime}+a_{m j} x_{j}^{\prime}\right) \equiv F\left(X_{1}+X_{1}^{\prime}\right. \\
& \left.X_{2}+X_{2}^{\prime}, X_{3}+X_{3}^{\prime}, \cdots, X_{m}+X_{m}^{\prime}\right)
\end{aligned}
$$

* Presented to the Society, August 29, 1929. 
where $X_{1}=a_{1 i} x_{i}^{\prime}, X_{1}^{\prime}=a_{1 j} x_{j}^{\prime}, X_{2}=a_{2 i} x_{i}^{\prime}, X_{2}^{\prime}=a_{2 j} x_{j}^{\prime}$, etc. We find the coefficient of $x_{i}^{\prime} x_{j}^{\prime n-r}$ in the group of terms*

$$
\begin{aligned}
\frac{1}{(n-r) !}\left(\frac{\partial F}{\partial X_{1}} X_{1}^{\prime}\right. & \left.+\frac{\partial F}{\partial X_{2}} X_{2}^{\prime}+\frac{\partial F}{\partial X_{3}} X_{3}^{\prime}+\cdots+\frac{\partial F}{\partial X_{m}} X_{m}^{\prime}\right)^{(n-r)} \\
\equiv & \frac{\left(x_{i}^{\prime} r x_{j}^{\prime}{ }^{n-r}\right)}{(n-r) !}\left(\frac{\partial F}{\partial a_{1 i}} a_{1 j}+\frac{\partial F}{\partial a_{2 i}} a_{2 j}\right. \\
& \left.+\frac{\partial F}{\partial a_{3 i}} a_{3 j}+\cdots+\frac{\partial F}{\partial a_{m i}} a_{m j}\right)^{(n-r)}
\end{aligned}
$$

where $\partial F / \partial a_{1 i}$ means $\partial F / \partial X_{1}$ with $X_{1}$ replaced by $a_{1 i}, X_{2}$ by $a_{2 i}, X_{3}$ by $a_{3 i}, \cdots, X_{m}$ by $a_{m i}$, and similarly for $\partial F / \partial a_{2 i}$, $\partial F / \partial a_{3 i}, \cdots, \partial F / \partial a_{m i}$. Hence we may conclude that a necessary and sufficient condition for the vanishing of the coefficient of $x_{i}^{\prime}{ }^{r} x_{j}^{\prime}{ }^{n-r}$ in the expansion of (3) is that the point $P_{j}\left(a_{1 j}, a_{2 j}, a_{3 j}, \cdots, a_{m \jmath}\right)$ shall lie on the $(n-r)$ th polar of $P_{i}\left(a_{1 i}, a_{2 i}, \cdots, a_{m i}\right)$ with respect to the locus of (1).

We obtain the coefficient of the term in $x_{i}^{\prime} x_{j}^{\prime} x_{k}^{\prime} \ldots$ $x_{l}{ }^{u} x_{p}^{\prime}{ }^{v}$ (where $r+s+t+\cdots+u+v=n$ ) in the expansion of (3) by the following device. We can write (3) as

(6) $F\left(Y_{1}+Y_{1}^{\prime}, Y_{2}+Y_{2}^{\prime}, Y_{3}+Y_{3}^{\prime}, \cdots, Y_{m}+Y_{m}^{\prime}\right)=0$,

where

$$
\begin{aligned}
Y_{1}= & a_{1 i} x_{i}^{\prime}, Y_{1}^{\prime}=a_{11} x_{1}^{\prime}+a_{12} x_{2}^{\prime}+a_{13} x_{3}^{\prime}+\cdots+a_{1 i-1} x_{i-1}^{\prime} \\
& +a_{1 i+1} x_{i+1}^{\prime}+a_{1 i+2} x_{i+2}^{\prime}+\cdots+a_{1 m} x_{m}^{\prime} \\
Y_{2}= & a_{2 i} x_{i}^{\prime}, Y_{2}^{\prime}=a_{21} x_{1}^{\prime}+a_{22} x_{2}^{\prime}+\cdots+a_{2 i-1} x_{i-1}^{\prime}+a_{2 i+1} x_{i+1}^{\prime} \\
& +\cdots+a_{2 m} x_{m}^{\prime}
\end{aligned}
$$

etc. We take the collection of terms

$$
\begin{aligned}
\frac{1}{(n-r) !}\left(\frac{\partial F}{\partial Y_{1}} Y_{1}^{\prime}\right. & +\frac{\partial F}{\partial Y_{2}} Y_{2}^{\prime}+\frac{\partial F}{\partial Y_{3}} Y_{3}^{\prime}+\cdots \\
& \left.+\frac{\partial F}{\partial Y_{m}} Y_{m}^{\prime}\right)^{(n-r)}
\end{aligned}
$$

* See Goursat-Hedrick Mathematical Analysis, vol. 1, pp. 107-108. For the Galois fields, see A. D. Campbell, The polar curves of plane algebraic curves in the Galois fields, this Bulletin, vol. 34 (1928), pp. 361-363. The methods of this paper may be readily generalized to the polars of an $n$-ic in $m$ variables. 
in the expansion of (6). All the terms with $x_{i}^{\prime r}$ as a factor must come from (7). We can write (7) in the form

$$
\begin{aligned}
& \frac{x_{i}^{\prime} r}{(n-r) !}\left(\frac{\partial F}{\partial a_{1 i}} Y_{1}^{\prime}+\frac{\partial F}{\partial a_{2 i}} Y_{2}^{\prime}+\frac{\partial F}{\partial a_{3 i}} Y_{3}^{\prime}+\cdots\right. \\
& \left.+\frac{\partial F}{\partial a_{m i}} Y_{m}^{\prime}\right)^{(n-r)} \text {. }
\end{aligned}
$$

If we equate (8) to zero we obtain the $(n-r)$ th polar of $P_{\imath}\left(a_{1 i}, a_{2 i}, a_{3 i}, \cdots, a_{m i}\right)$ with respect to the locus of (1). We can also write ( 7$)$ in the form

$$
\frac{x_{i}^{\prime} r}{(n-r) !} F^{\prime}\left(Y_{1}^{\prime}, Y_{2}^{\prime}, Y_{3}^{\prime}, \cdots, Y_{m}^{\prime}\right),
$$

where $F^{\prime}$ is a function of the $(n-r)$ th degree. We put (10) $Y_{1}^{\prime}=Z_{1}+Z_{1}^{\prime}, Y_{2}^{\prime}=Z_{2}+Z_{2}^{\prime}, \cdots, Y_{m}^{\prime}=Z_{m}+Z_{m}^{\prime}$, where

$$
\begin{aligned}
Z_{1}= & a_{1 j} x_{j}^{\prime}, Z_{1}^{\prime}=a_{11} x_{1}^{\prime}+a_{12} x_{2}^{\prime}+a_{13} x_{3}^{\prime}+\cdots+a_{1 i-1} x_{i-1}^{\prime} \\
& +a_{1 i+1} x_{i+1}^{\prime}+a_{1 i+2} x_{i+2}^{\prime}+\cdots+a_{1 j-1} x_{j-1}^{\prime}+a_{1 j+1} x_{j+1}^{\prime} \\
& +a_{1 j+2} x_{j+2}^{\prime}+\cdots+a_{1 m} x_{m}^{\prime}, Z_{2}=a_{2 j} x_{j}^{\prime},
\end{aligned}
$$

etc. Expanding (9), we find that all the terms in the expansion of (3) that have the factors $x_{i}^{\prime}$ r and $x_{j}^{\prime}{ }^{s}$ must be in the collection of terms

$$
\begin{gathered}
\frac{x_{i}^{\prime} r}{(n-r) !(n-r-s) !}\left(\frac{\partial F^{\prime}}{\partial Z_{1}} Z_{1}^{\prime}+\frac{\partial F^{\prime}}{\partial Z_{2}} Z_{2}^{\prime}+\frac{\partial F^{\prime}}{\partial Z_{3}} Z_{3}^{\prime}+\cdots\right. \\
\left.(11)+\frac{\partial F^{\prime}}{\partial Z_{m}} Z_{m}^{\prime}\right)^{(n-r-s)} \equiv \frac{\left(x_{i}^{\prime r} x_{j}^{\prime} s\right)}{(n-r) !(n-r-s) !}\left(\frac{\partial F^{\prime}}{\partial a_{1 j}} Z_{1}^{\prime}\right. \\
\left.+\frac{\partial F^{\prime}}{\partial a_{2 i}} Z_{2}^{\prime}+\frac{\partial F^{\prime}}{\partial a_{3 j}} Z_{3}^{\prime}+\cdots+\frac{\partial F^{\prime}}{\partial a_{m j}} Z_{m}^{\prime}\right)^{(n-r-s)}
\end{gathered}
$$

If we equate (11) to zero we shall have the $(n-r-s)$ th polar of the point $P_{\jmath}\left(a_{1 j}, a_{2 j}, a_{3 j}, \cdots, a_{m j}\right)$ with respect to the $(n-r)$ th polar of $P_{\imath}\left(a_{1 i}, a_{2 i}, a_{3 i}, \cdots, a_{m i}\right)$ with respect to the locus of (1). 
Next we take

$$
\begin{aligned}
& Z_{1}^{\prime}=W_{1}+W_{1}^{\prime}, Z_{2}^{\prime}=W_{2}+W_{2}^{\prime}, \\
& Z_{3}^{\prime}=W_{3}+W_{3}^{\prime}, \cdots, Z_{m}^{\prime}=W_{m}+W_{m}^{\prime}
\end{aligned}
$$

in (11), where

$$
\begin{aligned}
W_{1}= & a_{1 k} x_{k}^{\prime}, W_{1}^{\prime}=a_{11} x_{1}^{\prime}+a_{12} x_{2}^{\prime}+\cdots+a_{1 i-1} x_{i-1}^{\prime} \\
& +a_{1 i+1} x_{i+1}^{\prime}+\cdots+a_{1 j-1} x_{j-1}^{\prime}+a_{1 j+1} x_{j+1}^{\prime}+\cdots \\
& +a_{1 k-1} x_{k-1}^{\prime}+a_{1 k+1} x_{k+1}^{\prime}+\cdots+a_{1 m} x_{m}^{\prime}, W_{2}=a_{2 k} x_{k}^{\prime},
\end{aligned}
$$

etc. We repeat the above processes until we finally reach the collection of terms having all the factors $x_{i}{ }^{\prime}, x_{j}{ }^{\prime}$, $x_{k}{ }^{t}, \cdots, x_{l}{ }^{\prime}$, and $x_{p}{ }^{\prime}{ }^{v}$. Therefore, we see that for the coefficient of the term $x_{i}^{\prime}{ }^{r} x_{j}^{\prime}{ }^{s} x_{k}^{\prime}{ }^{t} \ldots x_{l}^{\prime}{ }^{u} x_{p}^{\prime}{ }^{v}$ in the expansion of (3) to vanish we must have the point $P_{p}\left(a_{1 p}, a_{2 p}, a_{3 p}, \cdots, a_{m p}\right)$ on the $(n-r-s-t-\cdots-u-v)$ th polar of $P_{l}\left(a_{1 l}, a_{2 l}, \cdots\right.$, $\left.a_{m l}\right)$ with respect to the $\cdots$ th polar of $\cdots, \cdots, \cdots$, with respect to the $(n-r-s-t)$ th polar of $P_{k}\left(a_{1 k}\right.$, $\left.a_{2 k}, \cdots, a_{m k}\right)$ with respect to the $(n-r-s)$ th polar of $P_{j}\left(a_{1 j}, a_{2 j}, \cdots, a_{m j}\right)$ with respect to the $(n-r)$ th polar of $P_{i}\left(a_{1 i}, a_{2 i}, \cdots, a_{m i}\right)$ with respect to the locus of (1).

It is noteworthy that this discussion applies to the ordinary complex or real domains and also to the Galois fields.

Syracuse UnIVERSITY 